\title{
Tailoring Supramolecular Nanotubes by Bile Salt Based Surfactant Mixtures**
}

\author{
Marta Gubitosi, Leana Travaglini, Maria Chiara di Gregorio, Nicolae V. Pavel, \\ José Vázquez Tato, Simona Sennato, Ulf Olsson, Karin Schillén, and Luciano Galantini*
}

\begin{abstract}
An approach for tailoring self-assembled tubular structures is described. By controlling the relative composition of a two-component surfactant mixture comprising the natural bile salt lithocholate and its bolamphiphilic derivative, it was possible to finely tune the nanotube cross-section of the mixed tubular aggregates that self-associated spontaneously in aqueous solution at $\mathrm{pH}$ 12. The diameter was found to vary up to $50 \%$ when the stoichiometric ratio of the two bile salts was changed. The tuning of supramolecular nanochannels with such remarkable precision is of significant interest for technological applications of these materials.
\end{abstract}

Tubular nanoscale structures are very attractive in the field of nanotechnology, where they can be used in a wide range of applications. ${ }^{[1]}$ In general, nanochannels can play specific roles according to their dimensions. For example, they can be used for selective encapsulation when their sizes are comparable to those of different biopolymers, organic or inorganic nanoparticles, viruses or DNA. ${ }^{[2]}$ The tailoring of the inner dimensions of the organic nanotubular structures is therefore crucial, and some efforts have already been made to control the diameter of the resultant assemblies through molecular design. ${ }^{[3]}$ Self-assembled organic nanotubes can be obtained from spontaneous association of several families of

[*] Dr. M. Gubitosi, Dr. L. Travaglini, Dr. M. C. di Gregorio, Prof. N. V. Pavel, Prof. L. Galantini

Dipartimento di Chimica, Sapienza Università di Roma

P.le Aldo Moro 5, 00185 Rome (Italy)

luciano.

E-mail: galantini@uniroma1.it

Prof. J. Vázquez Tato

Facultad de Ciencias, Universidad de Santiago de Compostela

Avda. Alfonso X El Sabio s/n, 27002 Lugo (Spain)

Dr. S. Sennato

Istitute of Complex Systems (ISC) - CNR, UOS Sapienza

P.le Aldo Moro 2, 00185 Rome (Italy)

Prof. U. Olsson, Prof. K. Schillén

Division of Physical Chemistry, Department of Chemistry

Center for Chemistry and Chemical Engineering

Lund University, 22100 Lund (Sweden)

[**] We thank Gunnel Karlsson, Marc Obiols-Rabasa, and Solmaz Bayati for valuable assistance with cryo-TEM, SAXS, and DSC. This work benefitted from the SasView software, developed by the DANSE project under NSF award DMR-0520547. We acknowledge financial support from the European Commission under the Seventh Framework Program by means of the grant agreement for the Integrated Infrastructure Initiative N. 262348 European Soft Matter Infrastructure (ESMI).

Dupporting information for this article is available on the WWW under http://dx.doi.org/10.1002/anie.201500445. compounds, ${ }^{[4]}$ for example, peptides,${ }^{[5]}$ bile acids,${ }^{[6]}$ phospholipids, ${ }^{[7]}$ polymers, ${ }^{[8]}$ glycolipids, ${ }^{[9]}$ and rationally designed amphiphiles. ${ }^{[10]}$ Particularly bile salts, thanks to their rigid structure ${ }^{[11]}$ have proven to be suitable as amphiphilic building blocks for such architectures. Their specific selfassembly behavior ${ }^{[12]}$ becomes enhanced in several bile salt derivatives. It has been demonstrated that they can form monodisperse hollow nanocylinders in aqueous solution, with various cross-section diameters ranging from several hundreds of nanometers down to only a few nanometers. ${ }^{[13]}$

Based on this knowledge, the present work has been dedicated to controlling the size (that is, the diameter of the cross-section) of supramolecular nanotubes by changing the composition of the surfactant mixtures. The presented systems are simple examples of how this is accomplished without resorting to a more complicated synthesis of designed molecules. The mixtures investigated within the scope of this study were constituted of a natural bile salt, namely sodium lithocholate (NaLC), and its bolaamphiphilic derivative NaManLC (see Figure 1).

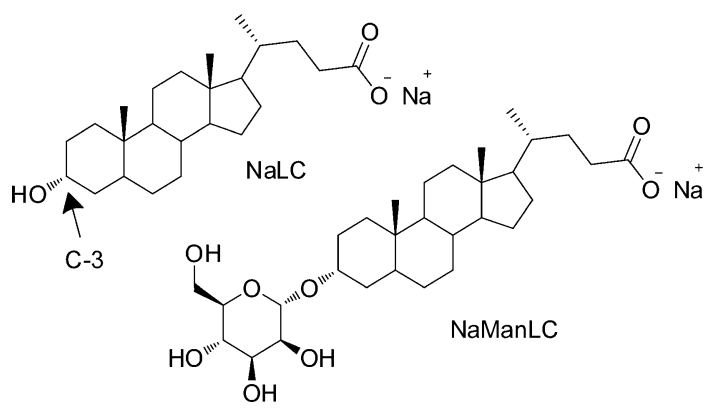

Figure 1. Molecular structures of $\mathrm{NaLC}$ and its derivative NaManLC, obtained by introducing a D-mannose unit into position C-3 of the steroidal backbone of the natural precursor.

Pure systems of NaLC as well as NaManLC form remarkably monodisperse hollow nanotubes in aqueous solution, but with different diameters (46 and $18.5 \mathrm{~nm}$ for NaLC and NaManLC, respectively). ${ }^{[14]}$ The objective of this study was thus to demonstrate that NaLC and NaManLC can form mixed aggregates and that it is possible to tune the nanotube diameter over a well-defined range in relative amounts. The ability of steroidal surfactants to form mixed tubules has been reported previously. ${ }^{[15]}$

Figure 2 a presents SAXS patterns of a representative collection of mixtures of NaLC and NaManLC at varying molar fractions of NaManLC, defined as $f_{\mathrm{NaManLC}}=$ 

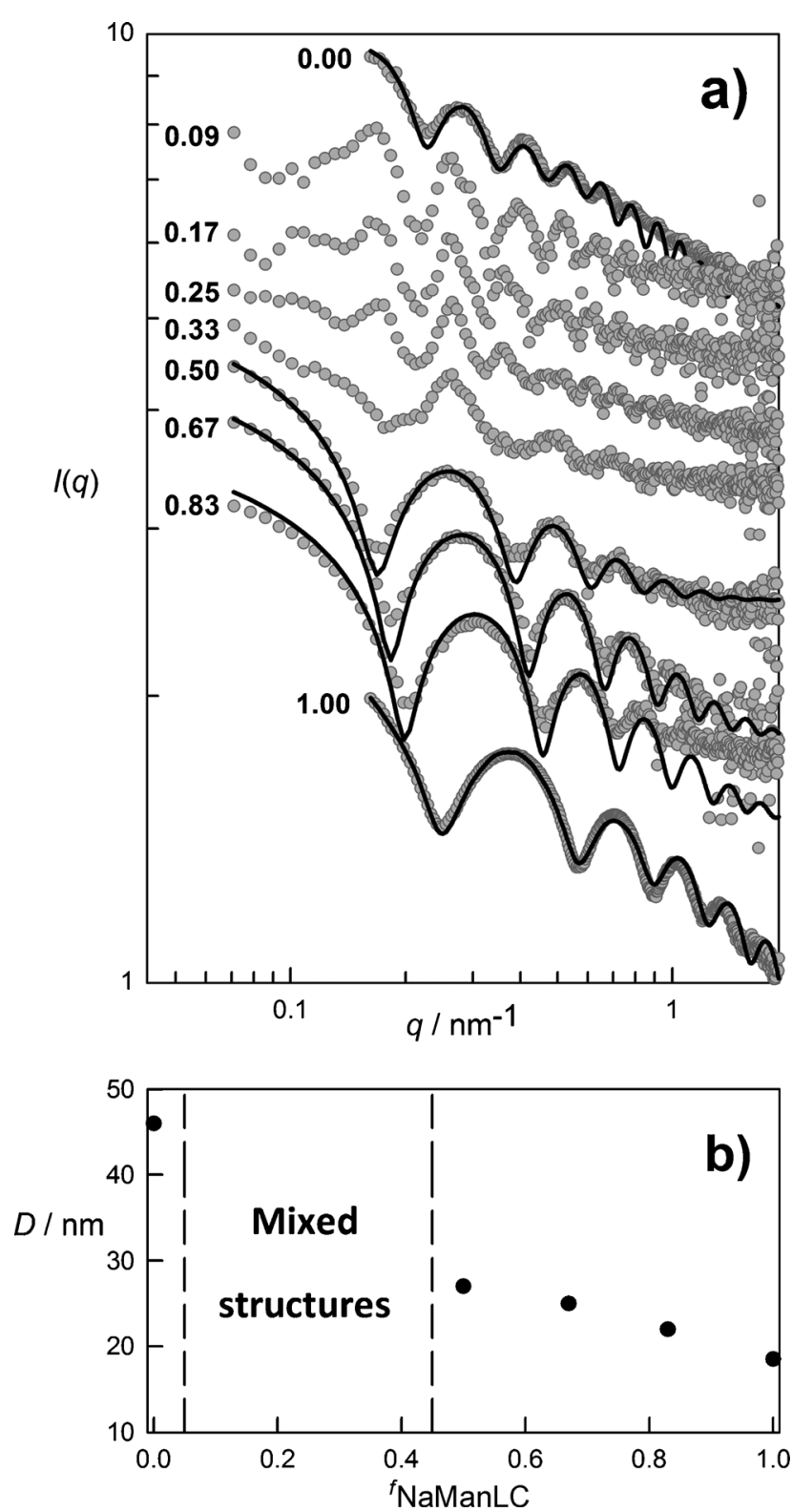

Figure 2. SAXS patterns and a partial phase map of NaManLC/NaLC mixtures equilibrated for two weeks. a) Experimental SAXS intensities $(\bullet)$ together with some model curves of hollow cylinders $(-)$ with cross-section diameters and thicknesses according to Table 1, at different molar fractions of NaManLC $\left(f_{\mathrm{NaManLC}}\right)$. For the sake of clarity, the curves have been shifted by convenient multiplicative factors. b) Partial phase map of the NaManLC/NaLC mixtures as a function of $f_{\text {NaManLC. }}$ The cross-section diameters of the tubules are reported for the samples with unimodal tubule distributions.

$[\mathrm{NaManLC}] /([\mathrm{NaManLC}]+[\mathrm{NaLC}])$, as well as of the two pure compounds at $20^{\circ} \mathrm{C}$. The scattering patterns for the two pure compounds showed regular and well-defined oscillations on a $q^{-2}$ decay, which corresponds to typical fingerprints for hollow nanotubes. As noticed, the positions of the maxima and minima varied owing to the different diameters of the tubules. When the two surfactants were mixed, two different scenarios were obtained depending on the fraction of NaManLC $\left(f_{\mathrm{NaManLC}}\right)$. Regular oscillating patterns could still be observed for all the NaManLC-rich mixtures up to the equimolar composition, that is, $f_{\mathrm{NaManLC}}=0.50$. However, the different maxima/minima demonstrated shifts towards lower $q$-values with a decreasing NaManLC fraction in the range from the pure derivative solution $\left(f_{\mathrm{NaManLC}}=1.00\right)$ to a mixed solution with $f_{\mathrm{NaManLC}}$ of 0.50 (Figure $2 \mathrm{~b}$ ). This behavior indicated that the diameter $D$ increased gradually as the amount of NaManLC decreased. The SAXS data obtained for these compositions were fitted with the model of hollow cylinders, and the diameters are presented in Table 1.

Table 1: Average diameter $D$ of the cross-section of hollow cylinder models used to fit the SAXS curves of the NaManLC/NaLC mixtures at different $f_{\mathrm{NaManLC}}{ }^{[\mathrm{a}]}$

\begin{tabular}{llllll}
\hline$f_{\text {NaManLC }}$ & 1.00 & 0.83 & 0.67 & 0.50 & 0.00 \\
$D[\mathrm{~nm}]$ & $18.5(8)$ & $22(1)$ & $25(1)$ & $27(1)$ & $46(2)$ \\
\hline
\end{tabular}

[a] The estimated standard deviations are given in parentheses.

It should be noted that the remarkable monodispersity of the diameter, reflected in well-resolved oscillations for the pure nanotubes, was maintained in the mixtures. The obtained thickness values, $t$, also considered as fit parameters, were in the range 1-2 $\mathrm{nm}$, which was in agreement with the expected length of the molecules. When increasing the NaLC fraction beyond the equimolar composition up to an $f_{\mathrm{NaManLC}}$ of 0.09 , irregular patterns were recorded. This suggests that other scattering structures or distributions of structures were present in these solutions.

WAXS patterns collected for some selected molar ratios (Figure S1 in the Supporting Information) show that, starting from the pure NaManLC nanotubes, increasing fractions of the $\mathrm{LC}^{-}$anions were included in the tubules, by adding NaLC up to equimolarity, which in turn induced increasing distortions in the molecular packing of the wall.

Cryo-TEM and AFM micrographs were recorded for two representative mixtures, i.e., $f_{\mathrm{NaManLC}}=0.50$ (Figure $3 \mathrm{a}-\mathrm{c}$ ) and 0.09 (Figure $3 \mathrm{~d}-\mathrm{f}$ ). Long and well-organized tubules with a high degree of monodispersity and average diameters of $30 \pm 1 \mathrm{~nm}$ were detected for the sample with $f_{\mathrm{NaManLC}}=0.50$ using cryo-TEM, see Figure $3 \mathrm{a}, \mathrm{b}$ and Figure $\mathrm{S} 2$ in the Supporting Information. The dimensions of the tubules were in good agreement with those obtained from the best fit of the SAXS data (Table 1). These images therefore provided further confirmation of a co-assembly of NaManLC and NaLC molecules into tubular structures. Such considerations can be reasonably extended to $f_{\mathrm{NaManLC}}=0.83$ and 0.67 , in which well-defined SAXS pattern oscillations allowed for fits using a hollow cylinder model. Monodisperse tubules were also imaged by AFM for the sample with $f_{\mathrm{NaManLC}}=0.50$ after drying on mica. The measured heights were significantly lower than the diameters inferred by SAXS and cryo-TEM, which suggests that the nanotubes lay flat on the support. Under this assumption, the obtained values of 4-5 nm were consistent with the nanotube wall thickness. Cryo-TEM and AFM images of the NaLC-rich samples $\left(f_{\text {NaManLC }}=0.09\right.$ and 0.25) showed a more complex distribution of structures (Figure $3 \mathrm{~d}-\mathrm{f}$ and Figure S3 in the Supporting Information). 


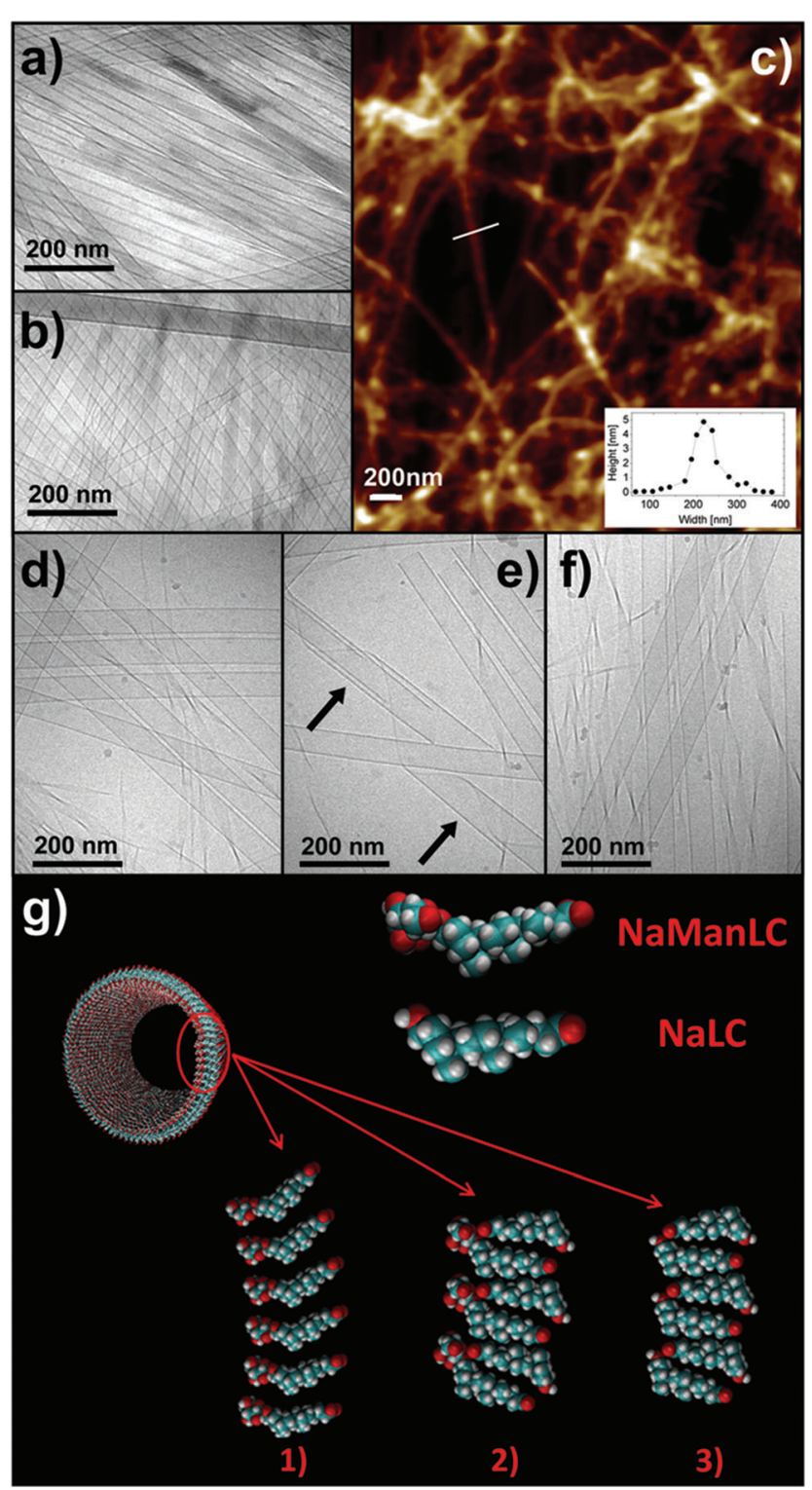

Figure 3. Micrographs from cryo-TEM $(a, b)$ and AFM (c) of samples at $f_{\mathrm{NaManLC}}=0.50$ and from cryo-TEM $(\mathrm{d}, \mathrm{e}, \mathrm{f})$ at $f_{\mathrm{NaManLC}}=0.09$ of

NaManLC/NaLC mixtures equilibrated for two weeks. The mixtures had a total concentration of $15.0 \mathrm{~mm}$ and $\mathrm{pH} 12.0$. The arrows indicate the presence of some helical structures. Possible aggregation models (g) for NaManLC (1), NaLC (3), and mixed (2) nanotubes. It has been hypothesized that NaManLC and NaLC molecules are organized in a parallel and antiparallel fashion, respectively. It is likely that some $\mathrm{NaLC}$ molecules can be introduced in an antiparallel manner in the NaManLC structure up to equimolar composition. Color code: $\mathrm{H}$ white, $\mathrm{C}$ cyan, $\mathrm{O}$ red.

We observed that bimodal distributions of tubular aggregates (that is, pure NaLC and equimolar NaManLC/NaLC nanotubes) were not consistent with the SAXS data (Supporting Information, Figure S4). Accordingly, cryo-TEM images showed that only wide tubules $(52 \pm 2 \mathrm{~nm}$ in diameter), probably formed by almost pure NaLC, were present together with some non-tubular aggregates (twisted or helical ribbons) in a very NaLC-rich sample, whereas no narrow tubules as those formed in the equimolar sample could be observed.
DSC measurements carried out on the samples at $f_{\mathrm{NaManLC}}$ of $1.00,0.67,0.50,0.33$, and 0.00 showed a single endothermic peak at each composition. Such peaks are related to the breaking of the observed structures at specific compositiondependent transition temperatures, suggesting that single or very similar mixed arrangements were formed in each mixture (Supporting Information, Figure S5a,b).

The complete data set from the SAXS, cryo-TEM and AFM experiments demonstrate that mixed tubes containing ManLC ${ }^{-}$and $\mathrm{LC}^{-}$anions were formed in the NaManLC/ $\mathrm{NaLC}$ mixtures in a wide range of compositions between the pure NaManLC and the equimolar ratio $\left(f_{\mathrm{NaManLC}}=0.50\right)$. The nanotubes were monodisperse with cross-sections that could be gradually regulated by changing the composition. The NaLC-rich mixtures exhibited a different aggregation behavior as revealed in the cryo-TEM images, since they displayed a variety of complex structures with separation of ribbons upon addition of very small fractions of NaManLC (Figure $3 \mathrm{~d}-\mathrm{f})$. It is known that the walls of supramolecular nanotubes can be characterized by an ordered (quasicrystalline) organization of the building blocks. ${ }^{[16]}$ Starting from a one-component tubule with such an ordered organization, the introduction of a different molecule, like in a mixture, is generally expected to determine a drastic molecular packing rearrangement, leading to the formation of a different supramolecular morphology (for instance a change from tubules to ribbons). Unlike general expectations, our results demonstrate that properly designed components can pack into mixed quasi-crystalline aggregates at various fractions with small lattice adjustments, thus allowing for the preparation of mixed tubules of varying sizes. We propose the following explanation for the selfassembly behavior of the NaManLC/NaLC mixtures in the scheme of Figure $3 \mathrm{~g}$. At first, it was assumed that pure NaManLC and NaLC tubules had two different supramolecular packings (in agreement with the WAXS data) and that the anions were arranged in parallel (NaManLC $)^{[14 c]}$ and antiparallel (NaLC) organizations. The arrangement within the $\mathrm{NaLC}$ nanotubes was based on molecular packings found in the structures of some crystals of lithocholate and other bile salts and was already proposed for helical ribbons and tubules of lithocholate-based systems. ${ }^{[17]}$ The model that we propose for the NaManLC nanotubes has some similarities with that proposed by Aggeli et al., ${ }^{[18]}$ with molecules stacked in a helical ribbon with the more hydrophilic moiety (the carboxylic group) located at the outer surface (see the Supporting Information). Conceivably, the sugar units are also involved in a stabilizing hydrogen bond network spread on the inner surface, as the formation of strong directional interactions (hydrogen bonds) is known to be fundamental for the stability of supramolecular nanotubes. ${ }^{[19]}$ These two arrangements correspond to different wall curvatures and diameters of pure NaManLC and NaLC tubules. Indeed, in the case of NaManLC tubules, the parallel crystalline arrangement with the hydrophilic charged groups of the molecules facing the bulk solution and the mannose groups directed towards the interior implies electrostatic repulsion between the charged groups leading to a lower curvature radius of its wall and a smaller cross-section. This type of 
packing of NaManLC molecules allows for the inclusion of lithocholate molecules with an antiparallel orientation, causing the introduction of carboxylic groups between the mannose moieties facing the interior of the tubule. This inclusion dictates the distancing of the sugar units while keeping almost constant the distances between the outer ManLC $^{-}$carboxylic groups, thus resulting in a less curved tubular wall and in an increased cross-section diameter. It also implies a weakening of the internal hydrogen bond network or its rearrangement due to the inclusion of the $\mathrm{LC}^{-}$ carboxylic groups. The higher the NaLC fraction in the mixture, the larger is the extent of this process, dictating a monotonic increase of the diameter in the mixed structure until the sample composition reaches a 1:1 molar ratio (that is, $\left.f_{\mathrm{NaManLC}}=0.50\right)$. On the other hand, the antiparallel organization of the $\mathrm{LC}^{-}$anions of the pure $\mathrm{NaLC}$ tubules does not permit the inclusion of ManLC $\mathrm{C}^{-}$anions, wherefore a separation of different aggregates occurs upon addition of small fractions of NaManLC to the pure NaLC samples. The pattern of the DSC transition temperatures as a function of composition (Supporting Information,Figure S5c) indicates that the thermal stability of the supramolecular arrangements decreases by changing from the parallel (pure NaManLC) to the antiparallel organization of $f_{\mathrm{NaManLC}}=0.50$, and that even less stable aggregates are formed in the NaLC-rich samples.

In summary, we have here described a novel approach to tune the nanotube cross-section size (up to $50 \%$ ) by controlling the stoichiometry of two-component surfactant mixtures. The results presented demonstrate that well-designed organic soft matter components can self-organize into mixed quasicrystalline aggregates at different fractions with minor lattice adjustments. This makes it possible to easily prepare mixed nanotubes with a range of different cross-sections, which will be tested for aligning and templating nanoparticles with selected sizes.

\section{Experimental Section}

Sample preparation: The derivative NaManLC was synthesized as described previously. ${ }^{[14 c]}$ NaManLC/NaLC mixtures were prepared at varying NaManLC fractions $\left(f_{\mathrm{NaManLC}}\right.$ from 0.09 to 0.83$)$ at a total concentration of $15.0 \mathrm{~mm}$ in $\mathrm{NaOH}$ aqueous solutions at $\mathrm{pH} 12.0$. Slightly turbid, viscous, and birefringent solutions were obtained for all the compositions after heating the samples to the boiling point and subsequently cooling them to room temperature.

Keywords: bile acids · nanochannels - nanotubes . self-assembly · tailoring

[1] a) H. Frusawa, T. Manabe, E. Kagiyama, K. Hirano, N. Kameta, M. Masuda, T. Shimizu, Sci. Rep. 2013, 3, 2165; b) R. Karlsson, M. Kurczy, R. Grzhibovskis, K. L. Adams, A. G. Ewing, A.-S Cans, M. V. Voinova, Nanoscale Res. Lett. 2011, 6, 421; c) D. Yoshida, K. Kim, I. Takumi, F. Yamaquchi, K. Adachi, A. Teramoto, Med. Mol. Morphol. 2013, 46, 86.
[2] A. de la Escosura, P. G. A. Janssen, A. P. H. J. Schenning, R. J. M. Nolte, J. J. L. M. Cornelissen, Angew. Chem. Int. Ed. 2010, 49, 5335; Angew. Chem. 2010, 122, 5463.

[3] M. Masuda, T. Shimizu, Langmuir 2004, 20, 5969.

[4] a) T. Shimizu, M. Masuda, H. Minamikawa, Chem. Rev. 2005, 105, 1401; b) D. T. Bong, T. D. Clark, J. R. Granja, M. Reza Ghadiri, Angew. Chem. Int. Ed. 2001, 40, 988; Angew. Chem. 2001, 113, 1016; c) T. Shimizu, H. Minamikawa, M. Kogiso, M. Aoyagi, N. Kameta, W. Ding, M. Masuda, Polym. J. 2014, 48, 831.

[5] C. Valéry, F. Artzner, M. Paternostre, Soft Matter 2011, 7, 9583.

[6] P. Terech, B. Jean, F. Ne, Adv. Mater. 2006, 18, 1571.

[7] J. M. Schnur, B. R. Ratna, J. V. Selinger, A. Singh, G. Jyothi, K. R. K. Easwaran, Science 1994, 264, 945.

[8] L. Cheng, G. Zhang, L. Zhu, D. Chen, M. Jiang, Angew. Chem. Int. Ed. 2008, 47, 10171; Angew. Chem. 2008, 120, 10325.

[9] K. Yoshida, H. Minamikawa, S. Kamiya, T. Shimizu, S. Isoda, J. Nanosci. Nanotechnol. 2007, 7, 960.

[10] a) Y.-A. Lin, A. G. Cheetham, P. Zhang, Y.-C. Ou, Y. Li, G. Liu, D. Hermida-Merino, I. W. Hamley, H. Cui, ACS Nano 2014, 8 , 12690; b) E. Lee, J.-K. Kim, M. Lee, Angew. Chem. Int. Ed. 2009, 48, 3657; Angew. Chem. 2009, 121, 3711.

[11] D. Madenci, S. U. Egelhaaf, Curr. Opin. Colloid Interface Sci. 2010, 15, 109.

[12] a) L. Galantini, E. Giglio, A. Leonelli, N. V. Pavel, J. Phys. Chem. B 2004, 108, 3078; b) S. Cozzolino, L. Galantini, E. Giglio, S. Hoffmann, C. Leggio, N. V. Pavel, J. Phys. Chem. B 2006, 110, 12351; c) L. Schefer, A. Sánchez-Ferrer, J. Adamcik, R. Mezzenga, Langmuir 2012, 28, 5999.

[13] a) N. Manghisi, C. Leggio, A. Jover, F. Meijide, N. V. Pavel, V. H. Soto Tellini, J. Vázquez Tato, R. G. Agostino, L. Galantini, Angew. Chem. Int. Ed. 2010, 49, 6604; Angew. Chem. 2010, 122, 6754; b) L. Travaglini, A. D'Annibale, K. Schillén, U. Olsson, S. Sennato, N. V. Pavel, L. Galantini, Chem. Commun. 2012, 48, 12011; c) M. C. di Gregorio, N. V. Pavel, A. Jover, F. Meijide, J. Vázquez Tato, V. H. Soto Tellini, A. Alfaro Vargas, O. Regev, Y. Kasavi, K. Schillén, K. L. Galantini, Phys. Chem. Chem. Phys. 2013, 15, 7560; d) F. Meijide, J. V. Trillo, S. de Frutos, L. Galantini, N. V. Pavel, V. H. Soto Tellini, A. Jover, J. Vázquez Tato, Steroids 2012, 77, 1205; e) L. Travaglini, et al., Phys. Chem. Chem. Phys. 2014, 16, 19492.

[14] a) X. Zhang, J. Zou, K. Tamhane, F. F. Kobzeff, J. Fang, Small 2010, 6, 217; b) B. Jean, L. Oss-Ronen, P. Terech, Y. Talmon, Adv. Mater. 2005, 17, 728; c) M. Gubitosi, L. Travaglini, A. D’Annibale, N. V. Pavel, J. Vázquez Tato, M. Obiols-Rabasa, S. Sennato, U. Olsson, K. Schillén, L. Galantini, Langmuir 2014, 30, 6358.

[15] X. Zhang, T. Bera, W. Liang, J. Fang, J. Phys. Chem. B 2011, 115, 14445.

[16] M. R. J. Vos, P. E. L. G. Leclère, H. Meekes, E. Vlieg, R. J. M. Nolte, N. A. J. M. Sommerdijk, Chem. Commun. 2010, 46, 6063.

[17] a) A. Pal, H. Basit, S. Sen, V. K. Aswalb, S. Bhattacharya, J. Mater. Chem. 2009, 19, 4325; b) S. Song, H. Wang, A. Song, J. Hao, Chem. Asian J. 2014, 9, 245; c) G. Briganti, A. A. D’Archivio, L. Galantini, E. Giglio, Langmuir 1996, 12, 1180.

[18] a) A. Aggeli, M. Bell, N. Boden, J. N. Keen, P. F. Knowles, T. C. Mcleish, M. Pitkeathly, S. E. Radford, Nature 1997, 386, 259; b) A. Aggeli, I. A. Nyrkova, M. Bell, R. Harding, L. Carrick, T. C. B. McLeish, A. N. Semenov, N. Boden, Proc. Natl. Acad. Sci. USA 2001, 98, 11857.

[19] J.-H. Fuhrhop, W. Helfrich, Chem. Rev. 1993, 93, 1565.

Received: January 16, 2015

Revised: February 26, 2015

Published online: 


\section{Communications}

Nanochannels

M. Gubitosi, L. Travaglini,

M. C. di Gregorio, N. V. Pavel,

J. Vázquez Tato, S. Sennato, U. Olsson,

K. Schillén, L. Galantini*

III-III

Tailoring Supramolecular Nanotubes by Bile Salt Based Surfactant Mixtures

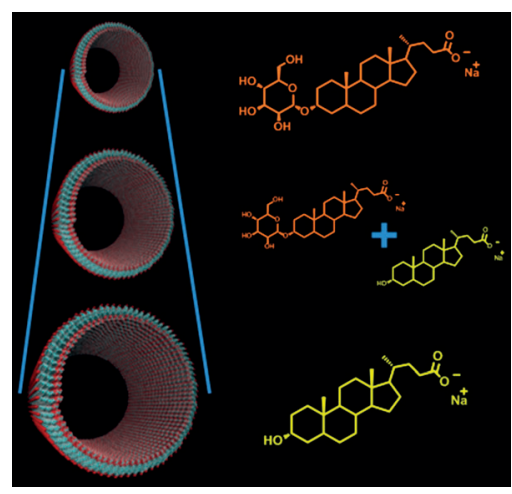

The perfect fit: The tailoring of selfassembled nanotube cross-sections is described for two-component mixtures of a bile acid and its derivative. By controlling the stoichiometry, diameters were found to vary by up to $50 \%$. Starting from pure tubules with a parallel arrangement of the monomer, a progressive inclusion of the second component in an antiparallel orientation is proposed to explain the variation of size. 